FINDINGS: This study is the first to isolate broadly reactive antibodies that are likely to protect against severe malaria in naturally immune individuals. Further characterization of antibody-antigen interactions will inform the development of this surface antigen as a vaccine candidate for malaria.

99529

\section{The Role of ATF6-Mediated Signaling in PARP Inhibitor Resistant Ovarian Cancer}

Alexandra and Benjamin Bitler

University of Colorado Anschutz Medical Campus

ABSTRACT IMPACT: This work has the potential to identify targetable pathways conveying resistance to PARP inhibitors that may improve ovarian cancer patient outcomes. OBJECTIVES/GOALS: High grade serous ovarian cancer is the deadliest gynecologic malignancy. PARP inhibitors are an FDA approved targeted therapy that is being used more and more frequently in the clinic. It is vital to understand mechanisms driving resistance to this therapy in order to develop treatments to improve patient responses. METHODS/ STUDY POPULATION: RNA-sequencing and transcription factor analysis was used to identify pathways of interest. An AP-1 transcriptional reporter assays was used to confirm results of the transcription factor analysis. An unbiased lentiviral shRNA screen was used to identify AP-1 subunits promoting PARP inhibitor resistance. Lentiviral transduction allowed for the knockdown ATF6. Comet assays and two-plasmid systems were used to determine levels of DNA damage and levels of DNA damage repair respectively. RESULTS/ANTICIPATED RESULTS: PARP inhibitor resistant cell lines have increased WNT signaling which promotes to increased DNA damage repair. PARP inhibitor resistant cell lines also have increased AP-1 transcriptional activity, ATF6 expression, and active p38. ATF6 knockdown and p38 inhibition is sufficient to resensitize cells to PARP inhibition. Upon treatment with PARP inhibitors, ATF6 knockdown as well as p38 inhibition lead to increased DNA damage in PARP inhibitor resistant cell lines. RNA-sequencing reveals a signifcant overlap in downregulated genes in cells treated with a $\beta$-catenin inhibitor and cells with an ATF6 knockdown. DISCUSSION/SIGNIFICANCE OF FINDINGS: Due to the increasing prevalence of PARP inhibitors in the clinic, it is vital to uncover mechanisms contributing to resistance. This work has the potential to identify targetable pathways conveying resistance to PARP inhibitors that may improve ovarian cancer patient outcomes.

\section{Precision Medicine}

\section{6}

\section{Deficiency of Novel Adipokine Tetranectin Increases Obesity and Insulin resistance in Females}

George Plasko ${ }^{1}$, Sijia He ${ }^{1}$, Jingjing Zhang ${ }^{2}$, Fen Liu $^{2}$, Juli Bai ${ }^{1}$, Lily Dong ${ }^{1}$ and Feng Liu ${ }^{1}$

${ }^{1}$ UTHSA and ${ }^{2}$ Central South University, Changsha, China

ABSTRACT IMPACT: Novel adipokines like tetranectin help explain why some people progress from obesity to diseases like diabetes, atherosclerosis, and dislipidemia OBJECTIVES/GOALS: Obesity has an established association with diabetes, dyslipidemia, and atherosclerosis.
Preventing progression from obesity to insulin resistance requires understanding of the regulatory mechanisms involved in the loss of insulin sensitivity. Adipose tissue is well known to function as an endocrine organ that produces many kinds of adipokines. METHODS/ STUDY POPULATION: Blood sample analysis from human patients and mice was used to determine associations between tetranectin and obesity. Samples were tested with a monoclonal anti-tetranectin antibody for detection with western blot. A tetranectin mutant knock out mouse line was compared to wild type littermates on high fat diet for 4 months. Insulin tolerance tests and glucose tolerance were used to determine progression to insulin resistance and glucose intolerance. Histological analysis of metabolic tissue was used to demonstrate adipocyte hypertrophy and liver steatosis. RESULTS/ANTICIPATED RESULTS: In the current study, we report the identification and initial characterization of a novel adipokine tetranectin. Tetranectin, which is coded by the C-type lectin domain family 3 member B (CLEC3B) gene, is ubiquitously expressed in various mouse tissues, whereas it is highly enriched in white adipose tissue. We found that the serum level of tetranectin was much higher in both obese and diabetic patients. Knocking out the tetranectin gene in mice protected against glucose intolerance in males but reduced insulin and glucose tolerance in females, without effects on food intake and body weight for either sex. Mechanistically, tetranectin targets liver tissues and its deficiency increases lipid accumulation in hepatocytes in females. DISCUSSION/SIGNIFICANCE OF FINDINGS: We have identified a novel adipokine which mediates a different metabolic crosstalk among tissues to maintain systemic glucose and lipid metabolism in different genders. Further investigation of tetranectin's function could yield a new target for precise therapeutic treatment for obesity and its associated metabolic diseases in different genders

73565

Defining tp53 tumor suppressor functions in zebrafish embryonal rhabdomyosarcoma*

Kunal Baxi, *Jiangfei Chen, Amanda Lipsitt, Nicole Hensch, Long Wang, Abhik Bandopadhyay, Aaron Sugalski, Andrea Gilbert, Eleanor Chen, Peter Houghton, Gail Tomlinson and Myron Ignatius University of Texas Health Science Center at San Antonio

ABSTRACT IMPACT: By assessing function of mutant (patient-specific) tp53 in zebrafish embryonal rhabdomyosarcoma will inform clinicians of the severity of mutant tp53 alleles. OBJECTIVES/GOALS: This study aims to define loss- and gain-of-function TP53 mutations by comparing effects in tp53-null and wild-type tumors. In addition, it aims to generate a rapid in vivo analysis platform to assign function to patient specific TP53 mutations in the clinic METHODS/STUDY POPULATION: To define tp53 function in ERMS pathogenesis, we previously generated a new tp53-null mutant (tp53-/-) in zebrafish by deleting the entire tp53 genomic locus using TALEN mutagenesis. tp53-/zebrafish spontaneously develop a spectrum of tumors including sarcomas, leukemia and germ cell tumors (Ignatius ... Baxi et. al., eLife) reminiscent of tumors observed in Trp53-null mice. Using the tp53-/mutants to generate kRASG12D-induced ERMS, we discovered that tp53 is a potent repressor of metastases but rather surprisingly had no effect on self-renewal (Ignatius ... Baxi et. al., eLife). Here, using tp53-/- zebrafish, we assessed effects of wild-type and mutant (patient specific) tp53 on tumor initiation, proliferation and apoptosis. RESULTS/ANTICIPATED RESULTS: ERMS tumor initiation in the tp53-/- background is observed in $>97 \%$ of animals whereas only $<40 \%$ of wild-type animals develop ERMS. Additionally, tp 53 is a potent suppressor of ERMS proliferation and its effect on apoptosis is minor. 